\title{
Viabilidade do uso de resíduos da agroindústria coureiro-calçadista no solo
}

\author{
Viability of using tannery and leather residues in the soil
}

\author{
Clesio Gianello $^{\mathrm{F}}$ Susan Carla Domaszak ${ }^{\mathrm{I}}$ Leandro Bortolon $^{\mathrm{I}}$ \\ Cláudio Henrique Kray ${ }^{I}$ Vanessa Martins ${ }^{I}$
}

\section{- NOTA -}

\section{RESUMO}

As indústrias couro-calçadistas geram grandes quantidades de resíduos que contém cromo, produto utilizado no curtimento do couro. Para avaliar os efeitos de três aplicações de lodo de curtume (LC), resíduo de rebaixadeira $(R R)$ e aparas de couro (AC) sobre o rendimento do sorgo (Sorghum graniferum) e rabanete (Raphanus sativus), foi conduzido um experimento em colunas de PVC, com três tipos de solo, durante o período de 1996 a 1999. Foram determinados no solo os valores de pH e o teor de cromo, bem como o teor de cromo nas partes comestíveis das plantas. Os resíduos não afetaram o crescimento das plantas, quando foram feitas a calagem e a adubação mineral. $O L C$ pode neutralizar a acidez do solo e suprir nitrogênio para as plantas. $O$ teor de cromo nas partes comestíveis das plantas aumentou com a aplicação sucessiva dos resíduos, estando, entretanto, sem exceder os teores aceitáveis para o consumo. Não foi observada a mobilidade de cromo para as camadas subsuperficiais do solo.

Palavras-chave: cromo, monitoramento, resíduo de curtume.

\section{ABSTRACT}

Tannery and leather industries generate high amounts of residues often containing chromium used as a tanning agent. To evaluate the effects of three applications of tannery sludge (TS), leather shavings (LS) and leather shreds (LS) on sorghum (Sorghum graniferum) and radish (Raphanus sativus), a microplot experiment with tree soil types was conducted during the 1996 to 1999 period. Soils pH and $\mathrm{Cr}$ contents in plants' edible parts were determined. The residues did not affect plant growth, when properly limed and fertilized. The TS can neutralize soil acidity and supply $N$ for the plants. Plant $\mathrm{Cr}$ contents in the edible parts increased with residues application, being however on the range appropriate for

\begin{abstract}
consumption. It was not observed $\mathrm{Cr}$ mobility to the soil subsurface layers.
\end{abstract}

Key words: chromium, monitoring, tannery residues.

Nas últimas décadas, têm-se observado maior conscientização da sociedade em relação à contaminação ambiental. A indústria coureiro-calçadista tem grande importância no Brasil, e o estado do Rio Grande do Sul (RS) destaca-se nesse ramo industrial. Muitos resíduos da indústria de curtumes são classificados como perigosos, devido ao seu alto teor de cromo. Entretanto, alguns resíduos de curtume possuem altos teores de matéria orgânica, de nutrientes e um elevado poder de neutralização da acidez dos solos. Essas características conferem poder fertilizante e corretivo a esses resíduos. Diversos estudos têm sido efetuados com o intuito de avaliar os impactos da aplicação dos resíduos de curtume em solos cultivados (CHANDRA et al., 2009; LOPÉZLUNAAet al., 2009; KRAY et al., 2008; GUPTA \& SINHA, 2007; FERREIRA et al., 2003). Além disso, têm sido estudados a dinâmica do cromo no solo e o efeito da aplicação dos resíduos no rendimento de culturas. $\mathrm{O}$ presente trabalho teve como objetivo avaliar o efeito imediato de várias aplicações de resíduos de curtume no rendimento do sorgo e do rabanete em alguns solos do RS, bem como os teores de cromo na planta e no solo.

Foram utilizados três solos do RS, pertencentes às unidades de mapeamento Arroio dos

'Universidade Federal do Rio Grande do Sul (UFRGS), Av. Bento Gonçalves, 7712, 91540-000, Porto Alegre, RS, Brasil. E-mail: cgianello@hotmail.com. *Autor para correspondência. 
Ratos (Plintossolo Argilúvico distrófico - FTd), Camaquã (Argissolo Vermelho-Amarelo distrófico PVAd) e Estação (Latossolo Vermelho distroférrico nitossólico - LVdf) (EMBRAPA, 1999), formados sobre os materiais de origem granito (FTd e PVAd) e basalto (LVdf). Esses solos apresentavam inicialmente as seguintes características principais, respectivamente: pHem água: 5,4, 5,2 e 5,4; teores de matéria orgânica $\left(\mathrm{em} \mathrm{g} \mathrm{kg}^{-1}\right): 22,0,24,0 \mathrm{e} 22,0$; teores de argila $\left(\mathrm{em} \mathrm{g} \mathrm{kg}^{-1}\right)$ 240,230 e 580 . Os teores de $\mathrm{P}$ e de $\mathrm{K}$ disponíveis eram baixos e altos para os três solos respectivamente (extraídos por solução Mehlich-1, conforme metodologia descrita por TEDESCO et al. (1995).

As unidades experimentais constaram de colunas de PVC que continham $36 \mathrm{~cm}$ de diâmetro e $60 \mathrm{~cm}$ de altura com volume de 60 litros. Os solos foram colocados nas colunas em duas camadas: uma camada superficial com os solos coletados na profundidade de zero a $20 \mathrm{~cm}$ e outra com os solos coletados na camada de 20 a $40 \mathrm{~cm}$, separados por uma tela de náilon. Para a drenagem, foi utilizada, no fundo das colunas, uma camada de brita com $5 \mathrm{~cm}$ de espessura, separada do solo por tela plástica (CASTILHOS, 1998). Na camada de zero a $20 \mathrm{~cm}$, foram aplicados os seguintes tratamentos: 1 - Testemunha (Test.); 2 - Adubação mineral + calagem $(\mathrm{NPK}+\mathrm{Ca}) ; 3$ - Adição de lodo de curtume + PK (LC1 + PK); 4 - Adição de resíduo de rebaixadeira + NPK + calcário $(\mathrm{RR}+\mathrm{NPK}+\mathrm{Ca}) ; 5$ Adição de aparas de couro + NPK + calcário (AC + $\mathrm{NPK}+\mathrm{Ca}$ ). A caracterização dos resíduos é dada na tabela 1. As quantidades dos resíduos adicionados são dadas na tabela 2, bem como a adubação mineral e o corretivo. O lodo de curtume foi adicionado para obter o mesmo valor de $\mathrm{pH}$ do tratamento 2 , com base no seu poder de neutralização. $\mathrm{O} R \mathrm{R}$ e as $\mathrm{AC}$ (pedaços de couro acabado, com um centímetro quadrado de área) foram adicionados em quantidades para obter aplicações semelhantes de $\mathrm{Cr}$ às aplicadas no tratamento 3 .

A primeira aplicação dos resíduos foi feita em junho de 1996, tendo sido cultivados trigo, alface e rabanete. Em novembro/97, foram reaplicados os mesmos resíduos, implantando-se a seguir a cultura de sorgo, o qual foi colhido em março/98, para determinação de rendimento de grãos. Em novembro/ 98, foi feita amostragem do solo das camadas superficial e subsuperficial, e cultivado rabanete, com a adubação conforme COMISSÃO... (1995), sendo feita a colheita de bulbos aos 30 dias. Em julho/99, foram novamente reaplicados os resíduos, sendo feita uma amostragem de solo da camada superficial em outubro/99 e implantando-se a cultura do rabanete, que foi colhido aos 30 dias. Nesse cultivo, os resíduos de RR e AC foram moídos até passar em peneira de $2 \mathrm{~mm}$ e aplicados ao solo. O experimento foi conduzido a céu aberto (com três repetições) e irrigação com água potável, na medida da exigência hídrica das plantas.

Após secagem $\left(\mathrm{a} 60^{\circ} \mathrm{C}\right)$ e quantificação, foi determinado o teor de $\mathrm{Cr}$ nos grãos de sorgo e nos bulbos de rabanete, por digestão com $\mathrm{HNO}_{3}-\mathrm{HClO}_{4}$ (TEDESCO et al., 1995), assim como os teores de $\mathrm{Cr}$ total no solo (seco a $40^{\circ} \mathrm{C}$ e tamisado em peneira de $2 \mathrm{~mm}$ ). $\mathrm{O}$ experimento foi conduzido em delineamento de blocos casualisados em arranjo fatorial (solos $\mathrm{x}$ tratamento). Foi feita a análise da variância e, quando os resultados de F mostraram-se significativos, os dados foram comparados com base na comparação múltipla de médias dos tratamentos pelo teste de Tukey ao nível de 5\% de probabilidade

Os resultados obtidos nos cultivos efetuados após a primeira aplicação dos resíduos foram apresentados por CASTILHOS (1998) e CASTILHOS et al. (2002). Os rendimentos de grão de sorgo (média de três solos) são apresentados na tabela 3. Pode-se observar que o rendimento determinado no tratamento testemunha foi muito baixo, devido à baixa fertilidade dos solos; nos demais tratamentos, os rendimentos foram semelhantes, indicando o potencial fertilizante em $\mathrm{N}$ do lodo de curtume. $\mathrm{O}$ mesmo foi observado na cultura do rabanete. Os teores de $\mathrm{Cr}$ nos grãos de sorgo aumentaram com a adição de lodo de curtume, assim como nos bulbos do rabanete (Tabela 3).

Tabela 1 - Principais características dos resíduos utilizados nos experimentos ${ }^{(1)}$.

\begin{tabular}{|c|c|c|c|c|c|c|c|}
\hline \multirow{2}{*}{ Resíduo } & \multirow{2}{*}{$\mathrm{pH}\left(\mathrm{H}_{2} \mathrm{O}\right)$} & $\mathrm{N}$ & $\mathrm{C}$ & $\mathrm{P}$ & $\mathrm{Ca}$ & $\mathrm{Cr}$ & $\mathrm{PN}^{(3)}$ \\
\hline & & \multicolumn{6}{|c|}{ - } \\
\hline 1- Lodo n.1 & 7,5 & 64 & 430 & 2,4 & 68 & 8,5 & 160 \\
\hline 2- Lodo n. $2^{(2)}$ & 8,9 & 23 & 210 & 3,3 & 55 & 27,4 & 210 \\
\hline 3-Resíduo de rebaixadeira & 3,8 & 140 & 370 & 0,3 & 1,6 & 17,1 & --- \\
\hline 4- Aparas de couro & 5,4 & 89 & 480 & 1,8 & 0,7 & 19,4 & --- \\
\hline
\end{tabular}

Ciência Rural, v.41, n.2, fev, 2011. 
Tabela 2 - Quantidades de calcário, adubos e resíduos adicionados ${ }^{(1)}$.

\begin{tabular}{|c|c|c|c|c|c|c|}
\hline \multirow[t]{2}{*}{ Solo } & \multirow{2}{*}{ Ano } & Lodo & $\begin{array}{l}\text { Resíduo de } \\
\text { rabaixadeira }\end{array}$ & Aparas de couro & Calcário $^{(2)}$ & \multirow{2}{*}{$\begin{array}{l}\text { Adubo mineral }^{(3)} \\
\text {----kg ha }{ }^{-1}----\end{array}$} \\
\hline & & \multicolumn{4}{|c|}{ 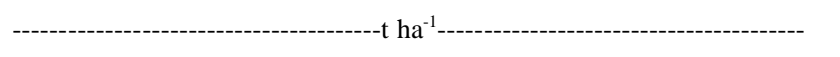 } & \\
\hline \multirow[t]{3}{*}{ FTd } & 1996 & 5,0 & 2,5 & 2,2 & 1,5 & $100+96+17$ \\
\hline & 1997 & 14,3 & 7,2 & 6,4 & 2,7 & $38+70+50$ \\
\hline & $1998 / 99$ & 18,6 & 18,3 & 19,4 & 3,2 & $85+134+238$ \\
\hline \multirow[t]{3}{*}{ PVAd } & 1996 & 8,1 & 4,0 & 3,6 & 2,0 & $100+70+34$ \\
\hline & 1997 & 18,1 & 9,1 & 8,1 & 2,7 & $37+114+95$ \\
\hline & 1998/99 & 14,5 & 15,2 & 16,1 & 3,0 & $84+241+353$ \\
\hline \multirow[t]{3}{*}{ LVdf } & 1996 & 8,8 & 4,4 & 3,8 & 2,0 & $100+40+34$ \\
\hline & 1997 & 20,8 & 10,3 & 9,1 & 2,7 & $48+281+82$ \\
\hline & $1998 / 99$ & 17,0 & 17,9 & 18,9 & 3,0 & $106+343+230$ \\
\hline
\end{tabular}

(1) Calculadas com base em $2 \times 10^{6} \mathrm{~kg} \mathrm{ha}^{-1}$ de solo seco.

(2) Mistura de $\mathrm{CaCO}_{3}+\mathrm{MgCO}_{3}$ (3:1) com PRNT 100\%, nas quantidades indicadas para atingir pH 6,0 nas aplicações de 1996 e 1997, e para pH 6,5 na aplicação de 1999.

(3) Valores de $\mathrm{N}, \mathrm{P}_{2} \mathrm{O}_{5}$ e $\mathrm{K}_{2} \mathrm{O}$, respectivamente, conforme as recomendações da (CFS RS/SC, 1995).

Com a aplicação de RR e de AC, entretanto, os teores de Cr nos grãos não aumentaram, indicando baixa translocação do metal para a parte aérea (e grãos) e/ou menor taxa de decomposição desses resíduos. Os teores de $\mathrm{Cr}$ determinados, tanto nos grãos de sorgo como nos bulbos do rabanete, situam-se na faixa considerada normal em plantas (KABATA-PENDIAS
\& PENDIAS, 1994), mesmo se os valores de Cr no solo estiverem nas classes de prevenção (testemunha, $\mathrm{NPK}+\mathrm{Ca}$ e AC) e intervenção (LC1 e RR), de acordo com os valores orientadores para solos proposto pela CETESB (CETESB, 2005).

Os valores de $\mathrm{pH}$ médios dos três solos, determinados na camada superficial em duas

Tabela 3 - Rendimento (média dos três solos) de grãos de sorgo e de matéria seca dos bulbos de rabanete cultivado em 1998 e 1999 e seus teores de $\mathrm{Cr}$ (médias de três repetições); valores médios de $\mathrm{pH}\left(\mathrm{em} \mathrm{H}_{2} \mathrm{O}\right)$ e teores médios de $\mathrm{Cr}$ total dos solos nas camadas superficial e subsuperficial, amostrados em duas épocas (média de três repetições).

\begin{tabular}{|c|c|c|c|c|c|c|}
\hline \multirow{2}{*}{ Tratamentos } & \multicolumn{2}{|c|}{-----------Sorgo----------- } & \multicolumn{2}{|c|}{--------Rabanete (98)-------- } & \multicolumn{2}{|c|}{--------Rabanete (99)-------- } \\
\hline & grãos & $\mathrm{Cr}$ & bulbos & $\mathrm{Cr}$ & Bulbos & $\mathrm{Cr}$ \\
\hline & $\mathrm{tha}^{-1}$ & $\mathrm{mg} \mathrm{kg}^{-1}$ & $\mathrm{~g} \mathrm{vaso}^{-1}$ & $\mathrm{mg} \mathrm{kg}^{-1}$ & $\mathrm{~g} \mathrm{vaso}^{-1}$ & $\mathrm{mg} \mathrm{kg}^{-1}$ \\
\hline T1- test. & $0,84 \mathrm{~b}$ & $0,11 \mathrm{c}$ & $0,07 \mathrm{~b}$ & & --- & $-{ }_{--}^{(1)}$ \\
\hline $\mathrm{T} 2-\mathrm{NPK}+\mathrm{Ca}$ & $7,35 \mathrm{a}$ & $0,71 \mathrm{~b}$ & $6,72 \mathrm{a}$ & $1,51 \mathrm{a}$ & $8,52 \mathrm{a}$ & $0,31 \mathrm{c}$ \\
\hline T3- LC1 + PK & $7,48 \mathrm{a}$ & $2,16 \mathrm{a}$ & $7,88 \mathrm{a}$ & $1,44 \mathrm{a}$ & $8,06 \mathrm{a}$ & $2,11 \mathrm{a}$ \\
\hline $\mathrm{T} 4-\mathrm{RR}+\mathrm{NPK}+\mathrm{Ca}$ & $7,55 \mathrm{a}$ & $0,62 \mathrm{~b}$ & $7,86 \mathrm{a}$ & $1,38 \mathrm{a}$ & $8,12 \mathrm{a}$ & $2,02 \mathrm{a}$ \\
\hline \multirow[t]{4}{*}{$\mathrm{T} 5-\mathrm{AC}+\mathrm{NPK}+\mathrm{Ca}$} & $6,99 \mathrm{a}$ & $0,55 \mathrm{~b}$ & $7,19 \mathrm{a}$ & $0,59 \mathrm{~b}$ & $8,09 \mathrm{a}$ & $1,22 \mathrm{bc}$ \\
\hline & \multicolumn{2}{|c|}{---------Valores de pH--------- } & \multicolumn{4}{|c|}{ 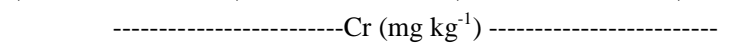 } \\
\hline & $11 / 98$ & & \multirow{2}{*}{\multicolumn{2}{|c|}{$\begin{array}{c}11 / 98 \\
\text { superficial }\end{array}$}} & \multicolumn{2}{|c|}{-----------10/99----------- } \\
\hline & \multicolumn{2}{|c|}{--------Camada superficial--------- } & & & \multirow{2}{*}{$\begin{array}{l}\text { subsuperficial } \\
64 \mathrm{a}\end{array}$} & \multirow{2}{*}{$\begin{array}{c}\text { superficial } \\
76 \mathrm{c}\end{array}$} \\
\hline T1- test. & $5,1 \mathrm{~b}$ & & \multicolumn{2}{|c|}{$68 \mathrm{c}$} & & \\
\hline $\mathrm{T} 2-\mathrm{NPK}+\mathrm{Ca}$ & $5,8 \mathrm{a}$ & & \multicolumn{2}{|c|}{$67 \mathrm{c}$} & $67 \mathrm{a}$ & $74 \mathrm{c}$ \\
\hline T3- LC1 + PK & $6,1 \mathrm{a}$ & & \multicolumn{2}{|c|}{$175 \mathrm{a}$} & $65 \mathrm{a}$ & $212 \mathrm{a}$ \\
\hline $\mathrm{T} 4-\mathrm{RR}+\mathrm{NPK}+\mathrm{Ca}$ & $5,8 \mathrm{a}$ & & \multicolumn{2}{|c|}{$109 \mathrm{~b}$} & $66 \mathrm{a}$ & $145 \mathrm{~b}$ \\
\hline $\mathrm{T} 5-\mathrm{AC}+\mathrm{NPK}+\mathrm{Ca}$ & $5,9 \mathrm{a}$ & & \multicolumn{2}{|c|}{$67 \mathrm{c}$} & 69 a & $78 \mathrm{c}$ \\
\hline
\end{tabular}

(1) Material insuficiente para análise. Médias seguidas pela mesma letra, na coluna, não diferem entre si pelo teste de Tukey (5\% de probabilidade).

Ciência Rural, v.41, n.2, fev, 2011. 
amostragens, são dados na tabela 3. Pode-se observar que tanto as adições de calcário como de lodo de curtume foram eficientes no aumento do $\mathrm{pH}$ até valores próximos aos desejados (5,9 na segunda e 6,8 na terceira aplicações dos resíduos), com base no poder de neutralização deles. A quantidade de lodo adicionada na terceira aplicação, entretanto, pode ter sido superestimada, atingindo o valor médio de 7,1. Devido ao curto período de tempo decorrido entre a aplicação e a amostragem do solo ( 3 meses), o valor de $\mathrm{pH}$ alto pode ser atribuído à menor taxa de reação do resíduo alcalino com o solo.

Os teores médios de $\mathrm{Cr}$ total da camada superficial dos solos aumentaram com as adições do LC e do RR. O aumento, entretanto, foi menor com a adição de AC, que são separadas pela peneira com $2 \mathrm{~mm}$, utilizado na preparação das amostras para a análise (Tabela 3). As quantidades de $\mathrm{Cr}$ contidas nas frações de resíduos que passam na peneira, portanto, são avaliadas como constituintes do solo, pois são solubilizadas pelos ácidos concentrados. A recuperação do $\mathrm{Cr}$ adicionado pelos resíduos (subtraindo-se os teores no tratamento testemunha) foi de 41,27 e $1 \%$ nos tratamentos com adições de LC (T3), RR (T4) e AC (T5), respectivamente. Baixas quantidades de $\mathrm{Cr}$ recuperado nas análises foram também observadas em outros trabalhos (FERREIRA et al., 2003). Elas foram atribuídas à separação do resíduo não decomposto durante o preparo da amostra e/ou à formação de compostos de grande estabilidade no solo. Nos tratamentos em que foram adicionados $\mathrm{RR}$ e AC finamente moídos, as recuperações do $\mathrm{Cr}$ foram de 77 e $42 \%$, respectivamente. Não foi observada translocação de $\mathrm{Cr}$ para a camada subsuperficial do solo (Tabela 3), da mesma forma que em estudo de campo (FERREIRA et al., 2003).

Os resíduos da indústria coureiro-calçadista não afetaram o crescimento das plantas, podendo ser fonte de $\mathrm{N}$ a elas. Os teores de $\mathrm{Cr}$ na parte comestível das plantas podem aumentar com a adição dos resíduos, não atingindo, entretanto, níveis tóxicos para o consumo. Não foi observada a translocação de $\mathrm{Cr}$ para a camada subsuperficial dos solos com a aplicação dos resíduos.

\section{REFERÊNCIAS}

CASTILHOS, D.D. Alterações químicas e biológicas devidas à adição de resíduos de curtume e de cromo hexavalente ao solo. 1998. 196f. Tese (Doutorado em Ciência do Solo) Programa de Pós-graduação em Ciência do Solo, Faculdade de Agronomia, Universidade Federal do Rio Grande do Sul, Porto Alegre, RS.

CASTILHOS, D.D. et al. Rendimentos de culturas e alterações químicas do solo tratado com resíduos de curtume e cromo hexavalente. Revista Brasileira de Ciência do Solo, v.26, p.1083-1092, 2002.
CETESB - Companhia de Tecnologia de Saneamento Ambiental. Caracterização das estações de rede automática de monitoramento da qualidade do ar na RMSP - estação Cerqueira César, 2005. Disponível em: <http://www.cetesb.sp.gov.br/ ar/relatorios/relatorios.asp>. Online. Acesso em: 20 dez. 2010.

CHANDRA, R. et al. Accumulation and distribution of toxic metals in wheat (Triticum aestivum L.) and Indian mustard (Brassica campestris L.) irrigated with distillery and tannery effluents. Journal of Hazardous Material, v.162, p.15141521,. 2009. Disponivel em: <http://dx.doi.org/10.1016/ j.jhazmat.2008.06.040>. Acesso em: 20 dez. 2010. doi: 10.1016/j.jhazmat.2008.06.040.

COMISSÃO DE FERTILIDADE DO SOLO RS/SC. Recomendações de adubação e calagem para os Estados do Rio Grande do Sul e Santa Catarina. 3.ed. Passo Fundo: SBCS/NRS, 1995. 224p.

DOMASZAK, S.C. Efeitos imediato e residual da aplicação de resíduos de curtume em plantas e solos. 2000. $107 \mathrm{f}$. Dissertação (Mestrado em Ciência do Solo) - Programa de Pósgraduação em Ciência do Solo, Faculdade de Agronomia, Universidade Federal do Rio Grande do Sul, Porto Alegre, RS.

EMBRAPA. Sistema brasileiro de classificação de solos. Brasilia: EMBRAPA - SPI, 1999. 412p.

FERREIRA, A.S. et al. Alterações de atributos químicos e biológicos de solo e rendimento de milho e soja pela utilização de resíduos de curtume e carbonífero. Revista Brasileira de Ciência do Solo, v.27, p.755-763, 2003. Disponível em: <http:/ www.scielo.br/scielo.php? script=sci_arttext\&pid $=$ S0 100 $06832003000400020 \& \operatorname{lng}=\mathrm{pt} \& \mathrm{nrm}=\mathrm{iso}>$. Acesso em: $20 \mathrm{dez}$. 2010. doi: $10.1590 / \mathrm{S} 0100-06832003000400020$.

GUPTA, A.K.; SINHA, S. Assessment of single extraction methods for the prediction of bioavailability of metals to Brassica juncea L. Czern. (var. Vaibhav) grown on tannery waste contaminated soil. Journal of Hazardous Material, v.149, p.144-150, 2007. Disponivel em: <http://dx.doi.org/ doi:10.1016/j.jhazmat.2007.03.062>. Acesso em: 20 dez. 2010. doi: 10.1016/j.jhazmat.2007.03.062.

KABATA-PENDIAS, A.; PENDIAS, H. Elements of group VI. In: _____. Trace elements in soils and plants. Boca Raton: CRC, 1994. Cap.11, 203-224p.

KRAY, C.H. et al. Tannery and coal mining residue disposal on soil. Revista Brasileira de Ciência do Solo, v.32, p.2877-2882, 2008. Disponível em: <http://www.scielo.br/ scielo.php ? script=sci_art text\&pid=S $0100-$ $06832008000700035 \& \operatorname{lng}=$ pt\&nrm=iso $>$. Acesso em: 20 dez. 2010. doi: 10.1590/S0100-06832008000700035.

LÓPEZ-LUNAA, J. et al. Toxicity assessment of soil amended with tannery sludge, trivalent chromium and hexavalent chromium, using wheat, oat and sorghum plants. Journal of Hazardous Material, v.163, p.829-834, 2009. Disponivel em: 〈http://dx.doi.org/ doi:10.1016/j.jhazmat.2008.07.034〉. Acesso em: 20 dez. 2010. doi:10.1016/j.jhazmat.2008.07.034.

TEDESCO, M.J. et al. Análises de solos, plantas e outros materiais. 2.ed. Porto Alegre: Departamento de solos, UFRGS, 1995. 174p. (Boletim Técnico, 5). 